Proceedings

\title{
Towards 3D Confocal Imaging with Laser-Machined Micro-Scanner ${ }^{\dagger}$
}

\author{
Hilmi Artun Oyman 1,2,*, Baris Can Efe 1, Mustafa Akin Icel 1, Yigit Daghan Gokdel 2, \\ Onur Ferhanoglu ${ }^{3}$ and Arda Deniz Yalcinkaya ${ }^{1}$ \\ 1 Department of Electrical \& Electronics Eng. Bebek, Bogazici University, 34342 Istanbul, Turkey; \\ can.efe@boun.edu.tr (B.C.E.); akin.icel@boun.edu.tr (M.A.I.); arda.yalcinkaya@boun.edu.tr (A.D.Y.) \\ 2 Department of Electrical \& Electronics Eng. Eyup, Istanbul Bilgi University, 34060 Istanbul, Turkey; \\ yigit.gokdel@bilgi.edu.tr \\ 3 Department of Electronics \& Communication Eng. Maslak, Istanbul Technical University, 34469 Istanbul, \\ Turkey; ferhanoglu@itu.edu.tr \\ * Correspondence: artun.oyman@boun.edu.tr; Tel.: +90-537-272-9669 \\ + Presented at the Eurosensors 2018 Conference, Graz, Austria, 9-12 September 2018.
}

Published: 23 November 2018

\begin{abstract}
A micro-scanner made of stainless-steel is fabricated via laser cutting technology for 3D Lissajous confocal imaging. The multi-gimbaled structure of the device provides two orthogonal torsional modes and three different out-of-plane modes. Torsional modes can be used to achieve 2D scan and all of the out-of-plane modes can be used in changing the focus of the micro-scanner to achieve a 3D scanning pattern. One of the out-of-plane modes along with two orthogonal torsional modes can be employed for scanning a large depth-stack in sparse fashion while another out-ofplane mode can satisfy a much higher scan fill-rate with less field of view (FOV). Simulations of the micro-scanner are obtained using finite element method (FEM) software and compared with the characterization data gathered from Laser Doppler Vibrometer (LDV). Using various out-of-plane modes, the constructed fill patterns are simulated on MATLAB and fill rates compared.
\end{abstract}

Keywords: laser-machined; confocal microscopy; micro-scanner; magnetic actuation; 3D imaging; Lissajous scan; stainless steel

\section{Introduction}

Among various optical imaging techniques, confocal microscopy draws attention as it provides high optical resolution and increased contrast [1]. Scanning the surface of the sample point by point with laser source while collecting the reflected light as out-of-focus light is eliminated with the help of a pinhole is called laser scanning confocal microscopy (LSCM). The scanner of the LSCM system plays a crucial role as the field of view (FOV), resolution and the frame rate are mainly defined by the structural material and topology of the scanner as well as the actuation technique. Stainless-steel scanner is previously fabricated with laser cutter [2] and 3D operation of a MEMS mirror is realized by using Lorentz force principle [3].

In this study we are focusing on 3D scanning micro-scanner fabricated out of stainless steel with high relative magnetic permeability (grade: 430 ) which can work in different out-of-plane modes. Laser cutting technology is preferred as fabrication technique since it provides rapid production, high resolution and high accuracy. The proposed scanner generates Lissajous scan pattern satisfying high fill rate. The mode frequencies of the micro-scanner are found using finite element simulations and verified using Laser Doppler Vibrometer. After comparison of simulation and LDV results, a detailed study is provided for different out-of-plane modes in simulation environment using MATLAB. 


\section{Mechanical Design}

Multi-gimbaled scanner is designed to work in different out-of-plane modes along with two orthogonal torsional modes for 3D scan (Figure 1a). In order to demonstrate the operation of the proposed device with different out-of-plane modes, the frequency of the first out-of-plane mode is made as low as possible by playing with the width of the outer flexures. Moreover, the displacement of this mode is also considered and increased to scan the whole sample area. The areas of the gimbals adjusted in a fashion that slow and fast scan modes are separated from the first out-of-plane mode. While the outer flexures controls the slow scan movement of the device, central flexures determines the fast scan. Moreover, inside flexures creates an additional out-of-plane mode with higher frequency to be used to scan a specified location on the sample. On the other hand, second out-ofplane mode is designed to have higher frequency to provide better fill ratio; hence, a more detailed scan. The dimensions and material properties of the proposed scanner are given in Table 1. In order to actuate the scanner, two permanent magnets are fixed close to the mirror with an inclination of 45 degrees to the fast axis scan to get maximum displacement for both slow and fast scan (Figure 1b).

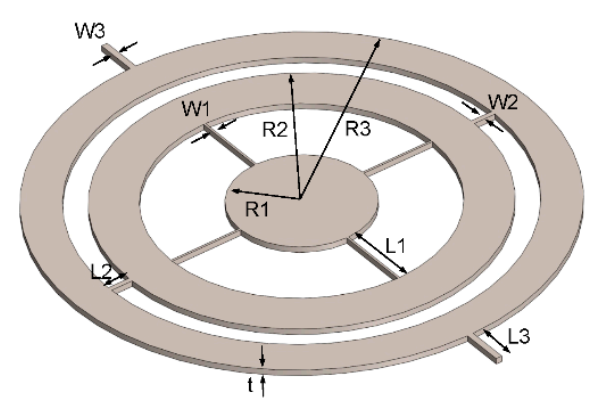

(a)

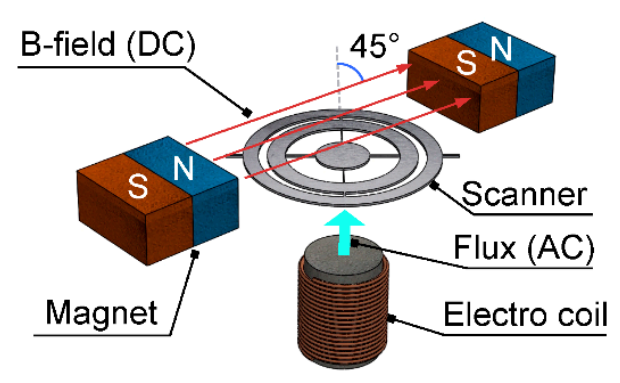

(b)

Figure 1. (a) Designed multi-gimbaled scanner. (b) The magnetic actuation principle of the scanner in terms of the interaction of the DC B-field generated by the permanent magnets and the AC flux induced by the external coil.

Table 1. Dimensions and material properties of the fabricated stainless-steel micro-scanner.

\begin{tabular}{lll}
\hline Parameter & Symbol & Value \\
\hline Radius of the mirror & R1 & $9 \mathrm{~mm}$ \\
Radius of the first gimbal & R2 & $12.5 \mathrm{~mm}$ \\
Radius of the second gimbal & R3 & $16.5 \mathrm{~mm}$ \\
Width of the inner flexures & W1 & $0.14 \mathrm{~mm}$ \\
Width of the center flexures & W2 & $0.32 \mathrm{~mm}$ \\
Width of the outer flexures & W3 & $0.41 \mathrm{~mm}$ \\
Length of the inner flexures & L1 & $5 \mathrm{~mm}$ \\
Length of the center flexures & L2 & $1.5 \mathrm{~mm}$ \\
Length of the outer flexures & L3 & $2.8 \mathrm{~mm}$ \\
Thickness of the device & $\mathrm{t}$ & $0.4 \mathrm{~mm}$ \\
Young's modulus & $\mathrm{E}$ & $200 \mathrm{GPa}$ \\
Density & $\mathrm{r}$ & $7740 \mathrm{~kg} / \mathrm{m}^{3}$ \\
Poisson's ratio & $\mathrm{n}$ & 0.28 \\
\hline
\end{tabular}

\section{Experimental Results}

Laser machining technology makes small patterns for a given material easier with high accuracy within a short amount of time. Fabrication process starts with the scanner design in a computeraided-design software. The proposed design is imported to the software of our custom laser-cutter which directs the focused beam on to the stainless-steel substrate and this process gives the structure its final shape via burning the edges. In order to generate a DC magnetic field, two permanent 
magnets are fixed close to the mirror with an inclination of 45 degrees to get maximum deflection for both slow and fast scan axes. As the frequency of the coil drive current is swept between 200-2600 $\mathrm{Hz}$ the deflection of the mirror is observed using a Laser-Doppler-Vibrometer (LDV) as given in Figure $2 b$. Resonance frequencies of the scanner are found by finite-element-method simulations and LDV results, show in good agreement and provided in Table 2.

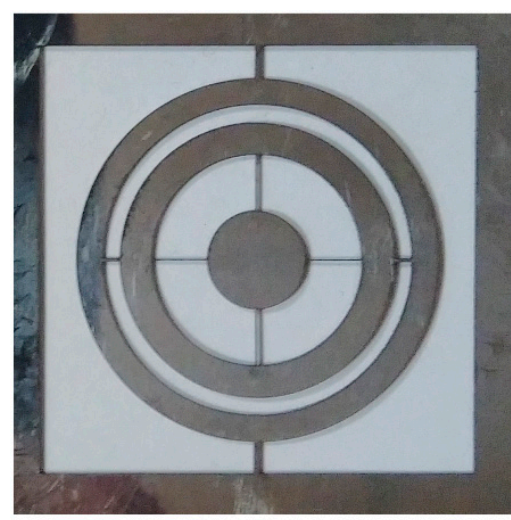

(a)

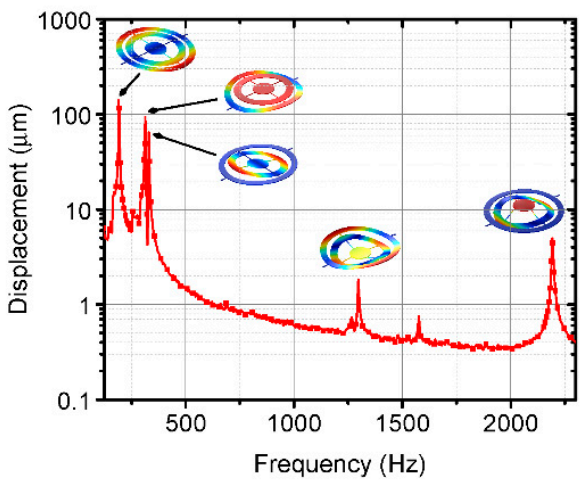

(b)

Figure 2. (a) Fabricated stainless-steel scanner. (b) Frequency response of the device. Exact frequency values of the corresponding modes are provided in Table 2.

Table 2. Comparison of mode frequencies (all in $\mathrm{Hz}$ ).

\begin{tabular}{lll}
\hline & FEM Results & LDV Results \\
\hline Slow Scan & 197 & 190 \\
Out-of-plane 1 & 315 & 313 \\
Fast Scan & 337 & 329 \\
Out-of-plane 2 & 1357 & 1296 \\
Out-of-plane 3 & 2196 & 2192 \\
\hline
\end{tabular}

\section{3D Scan Analysis}

3D scan analysis is performed to observe three dimensional scan performance of the designed scanner. For that purpose, out-of-plane 1 (slow) and out-of-plane 3 (fast) modes, given in Table 2, are chosen to investigate fill rates of considered volumes for slow and fast out-of-plane modes, respectively. A spherical solid with a radius of $15 \mu \mathrm{m}$ is considered as a target within a volume of $100 \mu \mathrm{m} \times 100 \mu \mathrm{m} \times 100 \mu \mathrm{m}$. Firstly, the whole volume is scanned with slow out-of-plane mode which has better displacement (Figure 2b). Scanned volume is considered as a 3D matrix $(36,36,36)$ which results in 36 slices along the $\mathrm{z}$-direction. Scan duration is set to $0.15 \mathrm{~s}$ for demonstration purpose, and fill rate of the target is found as $8 \%$ (Figure 3a). The corresponding axial slices for five different axial positions are given in Figure $3 \mathrm{~b}$. There are three different color seen on the slices such as white, blue and red. They represents unscanned, scanned and sphere unit cells, respectively. Then, intersection of blue and red unit cells is gathered to construct scanned unit cells of the sphere. The corresponding slices of constructed sphere is shown in Figure 3c. Thus, rough image of the target is detected by the scanner that operates in slow out-of-plane mode as shown in Figure 3d. Since the rough image of the sample obtained, this time the sample is scanned within a volume of $50 \mu \mathrm{m} \times 50 \mu \mathrm{m} \times 50 \mu \mathrm{m}$ with fast out-of-plane mode which has better fill ratio. As expected, fill rate of the target is increased to $25.7 \%$ for 70 the same scan duration as shown in Figure 3e. The corresponding axial slices for five different axial position are found as in Figure 3f,g and the reconstructed image is shown in Figure 3h. The axial positions are chosen as close as possible to the slow out-of-plane mode case. Furthermore, for a scan duration of $0.5 \mathrm{~s}, 24 \%$ and $69 \%$ fill rates are found for slow and fast out-of-plane mode, respectively. 


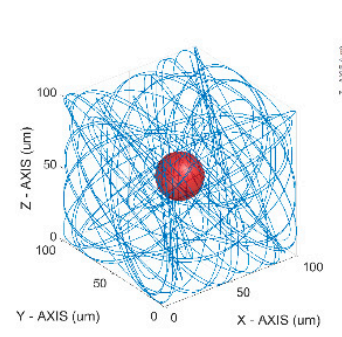

(a)

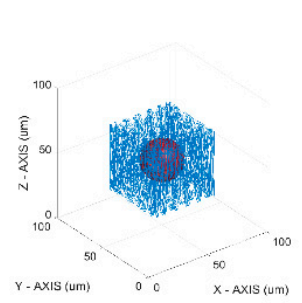

(e)

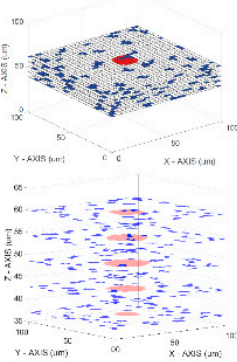

(b)

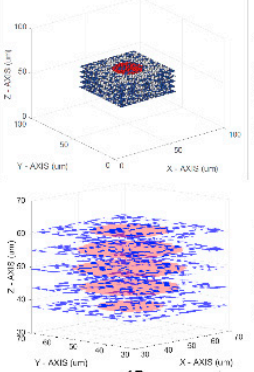

(f)

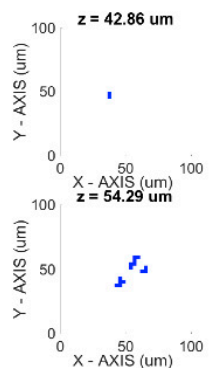

(c)

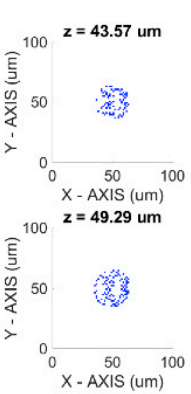

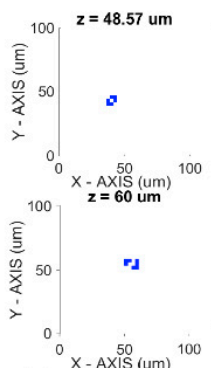

(c)

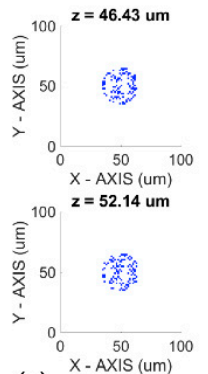

(g)

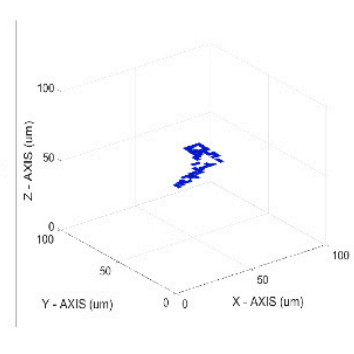

(d)

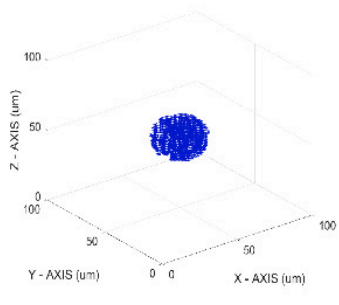

(h)

Figure 3. 3D Lissajous scan of a spherical solid with a radius of $15 \mu \mathrm{m}$ within a volume of $100 \mu \mathrm{m} \times$ $100 \mu \mathrm{m} \times 100 \mu \mathrm{m}$ for slow scan (a) and within a volume of $50 \mu \mathrm{m} \times 50 \mu \mathrm{m} \times 50 \mu \mathrm{m}$ for fast scan (e). Demonstration of $\mathrm{z}$ axis slices for slow (b) and fast scan (f). Detected pixels of five different slices for slow (c) and fast scan (g). Reconstructed image for slow (d) and fast scan (h).

\section{Conclusions}

A laser-machined stainless steel micro scanner is designed and manufactured to work in different out-of-plane modes for confocal microscopy. The proposed device accomplish a 3D Lissajous scan in a volume of $100 \mu \mathrm{m} \times 100 \mu \mathrm{m} \times 100 \mu \mathrm{m}$ to detect the sample by using its lowest outof-plane mode along with the torsional modes then it scans a reduced volume of $50 \mu \mathrm{m} \times 50 \mu \mathrm{m} \times 50$ $\mu \mathrm{m}$ with its highest frequency out-of-plane to get a sharper image. The main purpose of this work is to produce a low cost micro-scanner to be used in confocal microscopy setup for 3D imaging.

Acknowledgments: The authors acknowledge TUBITAK for the funding of project: TUBITAK SBAG 113S114, TUBA-GEBIP award.

Conflicts of Interest: The authors declare no conflict of interest.

\section{References}

1. Minsky, M. Confocal Scanning Microscope. Rapport Technique, US Patent 3,013,467, 19 December 1961.

2. Oyman, H.; Icel, M.; Efe, B.; Gokdel, Y.; Ferhanoglu, O.; Yalcinkaya, A. A Laser-Machined Stainless-Steel Micro-Scanner for ConfocalMicroscopy. Proceedings 2017, 1, 564.

3. Nagasawa, T.; Oguchi, Y.; Iwase, E. Operation of three-dimensional MEMS mirror by single superposed driving signal. Jpn. J. Appl. Phys. 2017, 56, 06GN12.

(c) 2018 by the authors. Licensee MDPI, Basel, Switzerland. This article is an open access article distributed under the terms and conditions of the Creative Commons Attribution (CC BY) license (http://creativecommons.org/licenses/by/4.0/). 